\title{
Location and multi-depot vehicle routing for emergency vehicles using tour coverage and random sampling
}

\author{
Alireza Goli* and Mehdi Alinaghian
}

Department of Industrial Engineering, Isfahan University of Technology, Isfahan, Iran

\begin{tabular}{l}
\hline C H R O N I C L E \\
\hline Article history: \\
Received January 29, 2015 \\
Received in revised format: \\
April 28, 2015 \\
Accepted May 5, 2015 \\
Available online \\
May 7 2015 \\
\hline Keywords: \\
Relief operations \\
Emergency system \\
Routing problem \\
Tour coverage \\
Random sampling
\end{tabular}

\section{A B S T R A C T}

\begin{abstract}
Distribution and optimum allocation of emergency resources are the most important tasks, which need to be accomplished during crisis. When a natural disaster such as earthquake, flood, etc. takes place, it is necessary to deliver rescue efforts as quickly as possible. Therefore, it is important to find optimum location and distribution of emergency relief resources. When a natural disaster occurs, it is not possible to reach some damaged areas. In this paper, location and multi-depot vehicle routing for emergency vehicles using tour coverage and random sampling is investigated. In this study, there is no need to visit all the places and some demand points receive their needs from the nearest possible location. The proposed study is implemented for some randomly generated numbers in different sizes. The preliminary results indicate that the proposed method was capable of reaching desirable solutions in reasonable amount of time.
\end{abstract}

\section{Introduction}

During the past few years, there have been several disaster incidents in the world and many people have died because no immediate help and rescue efforts were provided (Knott, 1987; Cao \& Lai, 2010). In the event of crises and catastrophes, time plays an essential role in development of providing humanitarian efforts (Croes, 1958; Feo \& Resende, 1989). The number of casualties and damage increases dramatically as time increases when a natural disaster occurs. Therefore, the logistics activities must be provided in minimum amount of time to help rescue people as quickly as possible. Disaster relief presents several unique logistics challenges, with issues including damaged transportation infrastructure, limited communication, and coordination of multiple agents (Kaufmann, 1975; Liu, 2004; Luis et al., 2012). Abounacer et al. (2014) considered a three-objective locationtransportation problem for disaster response. The planned to determine the number, the position and the mission of needed humanitarian aid distribution centers (HADC) within the disaster area. The

\footnotetext{
* Corresponding author.

E-mail address: a.goli@in.iut.ac.ir (A. Goli) 
transportation problem was associated with the distribution of aid from HADCs to demand points by considering three conflicting objectives. The first one minimized the total transportation duration of required products from the distribution centers to the demand points. The second one minimized the number of agents required to open and operate the selected distribution centers and finally, the last one minimized the non-covered demand for all demand points within the affected area. They proposed an epsilon-constraint technique for this problem and provided that it could construct the exact Pareto front. The results appeared to show that the computing time needed by the proposed method could be large for some instances and a heuristic method was developed for good approximation of the Pareto front in reasonable amount of computing times. Afshar and Haghani (2012) developed a model, which described the integrated logistics operations associated with natural disasters. They proposed a mathematical model, which control the flow of various relief commodities from the sources through the supply chain and until they were delivered to the hands of recipients. The structure of the network was in compliance with FEMA's complex logistics structure. The proposed model considered different issues such as vehicle routing and pick up or delivery schedules as well as finding the optimal locations for various layers of temporary facilities. The proposed model provided the opportunity for a centralized operation plan, which could eliminate delays and assign the limited resources to the best possible use. Barbarosoğlu et al. (2002) developed a mathematical model for helicopter mission planning during a disaster relief operation. Doerner et al. (2007) presented a multi-objective combinatorial optimization (MOCO) formulation for a location-routing problem in healthcare management. Ghaffari-Nasab et al. (2013) considered the location-routing problem with fuzzy demands (LRPFD), which could arise in many real-world situations in logistics management, and a fuzzy chance constrained program was designed to model it, based on the fuzzy credibility theory.

\section{The proposed study}

In this paper, location and multi-depot vehicle routing for emergency vehicles using tour coverage and random sampling is investigated. In this study, there is no need to visit all the places and some demand points receive their needs from the nearest possible location. In this paper, demand is considered as uncertain parameters and we use fuzzy numbers to handle uncertainty as follows,

$$
\mu_{\tilde{D}}(x)= \begin{cases}\frac{\left(x-d_{1}\right)}{\left(d_{2}-d_{1}\right)} & d_{1} \leq x \leq d_{2} \\ 1 & x=d_{2} \\ \frac{\left(d_{3}-x\right)}{\left(d_{3}-d_{2}\right)} & d_{2} \leq x \leq d_{3} \\ 0 & \text { otherwise }\end{cases}
$$

Here $\mu_{\tilde{D}}(x)$ is a membership function of demand $D$ at point $x$, which is defined in terms of trapezoid number. According to Eq. (1), customer's demand is between $d_{1}$ and $d_{3}$, therefore, the possibility (Pos), necessity ( $\mathrm{Nec}$ ) and credibility $(\mathrm{Cr})$ of demand are defined as follows,

$$
\begin{aligned}
& \operatorname{Pos}\{\tilde{D} \geq r\}= \begin{cases}1 & \text { if } r \leq d_{2} \\
\frac{d_{3}-r}{d_{3}-d_{2}} & \text { if } d_{2} \leq r \leq d_{3} \\
0 & \text { if } r \geq d_{3}\end{cases} \\
& \operatorname{Nec}\{\tilde{D} \geq r\}= \begin{cases}1 & \text { if } r \leq d_{1} \\
\frac{d_{2}-r}{d_{2}-d_{1}} & \text { if } d_{1} \leq r \leq d_{2} \\
0 & \text { if } r \geq d_{2}\end{cases}
\end{aligned}
$$




$$
\operatorname{Cr}\{\tilde{D} \geq r\}= \begin{cases}1 & \text { if } r \leq d_{1} \\ \frac{2 d_{2}-d_{1}-r}{2\left(d_{2}-d_{1}\right)} & \text { if } d_{1} \leq r \leq d_{2} \\ \frac{d_{3}-r}{2\left(d_{3}-d_{2}\right)} & \text { if } d_{2} \leq r \leq d_{3} \\ 0 & \text { if } r \geq d_{3}\end{cases}
$$

In this paper, to estimate demand, we use credibility function. In other words, given a credibility value, we estimate demand routing, which is determined based on the existing possibilities. An optimal value of credibility plays essential role for optimum routing system. In case, demand is over estimated, routing vehicle must return to depot with empty capacity and reschedule its trip. In addition, when demand is underestimated, it is possible to miss some demand, which increases the response time. The following algorithm determines optimum level of demand.

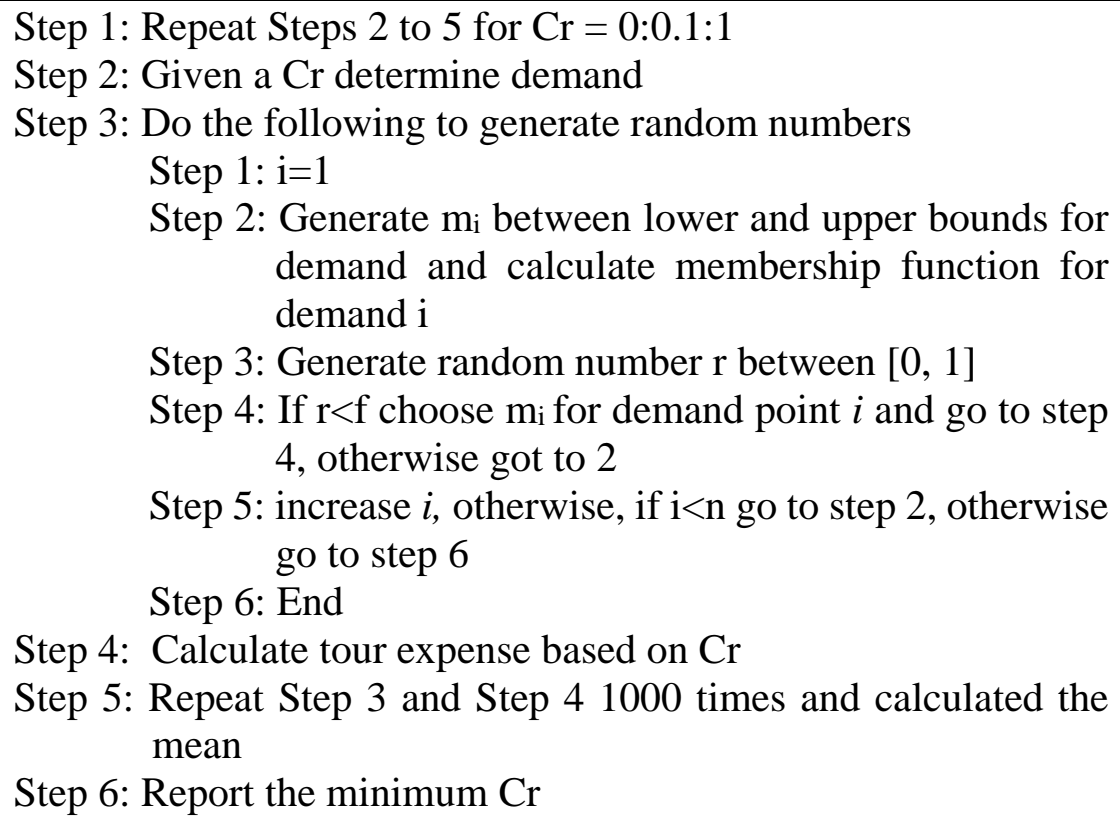

Step 4: Calculate tour expense based on $\mathrm{Cr}$

Step 5: Repeat Step 3 and Step 41000 times and calculated the mean

Step 6: Report the minimum $\mathrm{Cr}$

In this paper, an integrated model to locate temporary relief centers in the affected area, allocation and routing of damage to the goods required by these centers is offered. For all disaster relief operations, an immediate action is needed for the affected people for providing them necessary medical products. Therefore, all routes are considered as open routes and the primary objective is to minimize the total service time. All emergency facilities can be located inside or in the vicinity of the damaged units. Therefore, the services are divided into direct or coverage system. In other words, any service can be either delivered directly or placed in a place near the accident so that others can finish the operations. This type of service delivery is more suitable for rural areas facing natural disasters such as the earthquakes, fire, etc. is considered with negligible cost of establishment. There is also some main depots to provide necessary items required for these units. We assume there are some vehicles inside the main depots at time zero to provide necessary requirements such as drugs for other small depots.

Sets:

$R^{\prime} \quad$ Set of all possible points $R^{\prime}=\{1,2, \ldots P, P+1, P+2, \ldots R, R+1\}$ where $R+1$ is a dummy point

$\mathrm{Ba} \quad$ Set of all possible points, which is not possible to have relief agency.

$K^{\prime} \quad$ Set of all possible vehicles with $K^{\prime}=\{1,2, \ldots, \mathrm{K}\}$

pet of main depots $p=\{1,2, \ldots, P\}$ 


\section{Parameters:}

$t_{i j} \quad$ Travel time between node $i$ to node $j$

$\tilde{d}_{i} \quad$ Fuzzy demand for point $i$

$\operatorname{dis}_{\max }$ Maximum cover radius

$a_{i j} \quad$ A binary parameter, which is one of distance between node $i$ and $j$ is less than $d i s_{\max }$, and zero, otherwise

$v_{k p} \quad$ A binary parameter, which is one if vehicle $p$ exits at point $k$ at time zero

$Q \quad$ Capacity of vehicle

$M \quad$ A big number

Decision variables:

$x_{i j k} \quad$ A binary variable, which is one if route $i-j$ is covered by vehicle $k$, and zero, otherwise

$C D_{i} \quad$ Accumulated demand at point $i$

$y_{i} \quad$ A binary variable, which is one if point $i$ is covered by a truck and zero, otherwise

$\mathrm{D}_{\mathrm{ij}} \quad$ A binary variable, which is one of demand for point $i$ is assigned to point $j$

$u_{i k} \quad$ Time that vehicle $k$ reaches to $i$

$Z \quad$ Time of delivering goods to the last demand point

\section{Mathematical model}

$\min Z=\max _{k}\left\{u_{(R+1) k}\right\}$

Subject to

$\sum_{\substack{k=1 \\ l \neq P+1 \\ l \neq m}}^{K} x_{l m k}=y_{m} \quad m=2,3, \ldots, R$

$\sum_{m=2}^{R} x_{p m k}=v_{k p} \quad k=1,2, \ldots, K \quad p=1,2, \ldots, p$

$\sum_{k=1}^{K} \sum_{i=1}^{R+1} x_{i 1 k}=0$

$\sum_{i=2}^{R} X_{i(R+1) k}=1 \quad \forall k \in K$

$\sum_{k=1}^{K} \sum_{j=1}^{R+1} x_{(R+1) \mathrm{jk}}=0$

$\sum_{m=1}^{R+1} x_{m l k}-\sum_{m=1}^{R+1} x_{l m k}=0 \quad l=P+1,3, \ldots, R \quad k=1,2, \ldots K$

$\sum_{j=2}^{R} a_{i j} D_{i j}=1 \quad \forall i \in n$

$y_{i} \leq 1-\sum_{\substack{l=2 \\ l \neq i}}^{R} D_{i l} \quad i=1,2, \ldots, n$ 


$$
\begin{aligned}
& \sum_{i=2}^{R} \tilde{d}_{i} D_{i j}=C D_{j} \quad \forall j=2, \ldots, R \\
& \sum_{i=2}^{R} \sum_{j=2}^{R+1} C D_{i} x_{i j k} \leq Q \quad \forall k \in K \\
& y_{i}=D_{i i} \quad i=2, \ldots, R \\
& \sum_{i \in B a} y_{i}=0 \\
& \sum_{k=1}^{K} u_{P k}=0 \quad p=1,2, \ldots, P \\
& u_{i k}+t_{i j} \leq u_{j k}+M\left(1-x_{i j k}\right) \quad \forall i=1, \ldots, R \quad j=P+1, \ldots, R+1 \quad k \in K \\
& x_{i j k}, y_{i}, D_{i j} \in\{0,1\} \quad i, j \in R, k \in K \\
& C D_{i}, u_{i k} \geq 0 \quad i \in R, k \in K
\end{aligned}
$$

Eq. (6) specifies that a vehicle only covers temporary stations. Eq. (7-8) guarantee that all vehicles are used in operations and they do not go back to their origins. Eqs. (9-10) guarantee that all vehicles must be connected to dummy depot $R+1$ and no vehicle is permitted to exist from this depot. Eq. (11) is used to make sure that once a vehicle is entered into a node it will exit for sure. Eq. (12) and Eq. (13) are used to assign possible demands of some nodes to their neighbors covered by. Eq. (14) and Eq. (15) are associated with capacity of vehicles. According to Eq. (16) if there is a demand for a point $i$ it will not receive it from other points. Eq. (17) assures that temporary depots are not located in undesirable points. Eq. (18) and Eq. (19) are considered for removing sub-tour and finally Eq. (20) and Eq. (21) determine the type of variables. In this paper, we use a dummy variable $R+1$ to calculate the time trips and we assume that all vehicles must go to node $R+1$ with zero time. As we can see, Eq. (5) and Eq. (15) are nonlinear and we use the following equations to linearize them.

$\min \mathrm{Z}$

$$
\begin{aligned}
& Z \geq u_{(R+1) k} \quad \forall k \in K \\
& \sum_{l=2}^{R} \sum_{m=1}^{R+1} x_{l m k}^{\prime} \leq Q_{k} \quad k=1,2, \ldots, K \\
& x_{l m k}^{\prime} \leq M x_{l m k} \quad l=P+1, \ldots, R \quad m=1,2, \ldots, R+1 \quad k=1,2, \ldots, K \\
& x_{l m k}^{\prime} \leq C D_{l} \quad l=2,3, \ldots, R \quad m=1,2, \ldots, R+1 \quad k=1,2, \ldots, K \\
& x_{l m k}^{\prime} \geq C D_{l}-M\left(1-x_{l m k}\right) \quad l=P+1, \ldots, R \quad m=1,2, \ldots, R+1 \quad k=1,2, \ldots, K
\end{aligned}
$$

The resulted mixed integer programming can be solved for optimality for small sized problems. However, as the size of the problem increases, the problem can be time consuming and therefore, we propose metaheuristics; namely Variable Neighborhood Search (VNS) (Fleszar et al., 2009) to solve the resulted problem. VNS was first developed by Mladenović and Hansen (1997) and first it develops 
several initial solution and then it improves the resulted solution. When it is not possible to improve the existing optimal solution, the algorithm expands the existing structure. Consider $N_{l}$ with $l=\left\{1,2, \ldots, l_{\max }\right\}$, which is structure of existing neighborhood and $N_{l}(x)$ is the set of neighborhood $x$ with $N_{l}$. The algorithm has two phases of shake and local search. The proposed VNS uses two strings to generate the solution. Fig. 1 shows the first string

\begin{tabular}{|l|l|l|l|l|l|l|}
\hline 1 & 1 & 0 & 1 & 0 & 0 & 1 \\
\hline
\end{tabular}

Fig. 1. The first string visiting and transition nodes

In Fig. 1, each node with a value of one represents a visiting node and a node with zero value represents a transition node. In this figure, nodes 1, 2, 4 and 7 are visiting nodes and nodes 3, 5 and 6 are transition nodes. The second string demonstrates the vehicle and routing. For example, Fig. 2 shows a sample in line with Fig.1.

\begin{tabular}{|l|l|l|l|l|l|l|l|l|}
\hline 1 & 4 & 3 & 0 & 1 & 2 & 5 & 7 & 6 \\
\hline
\end{tabular}

Fig. 2. The second string for route and vehicle

According to Fig. 2 and based on the information of Fig. 1, first vehicle starts from node 1 and visits node 4 and node 3 , which is a transition node and receives its service from node 4 . The second vehicle also starts from node 1 and visits nodes 2 and 7, respectively. Nodes 5 and 6 are covered by nodes 2 and 7 depending on their distances. The proposed study of this paper uses two methods of serial and parallel for generating initial solutions. In both methods, the proposed study generates 1000 randomly generated solutions and then it chooses the best ones as candidates. Both algorithms use an initial step where all vehicles start trip from the main depot and calculates the following

$$
\text { ratio }_{j k}=\frac{\mathrm{D}}{(C P)^{v}} \text {, }
$$

where $D$ is the distance of node $j$ from the last visiting node by vehicle $k$ and $C P$ is the number of point in the vicinity of node $j$. We consider a value of 0.5 for $v$, which appears to be appropriate method, empirically.

\subsection{Serial operations}

The serial operations consists of the following steps,

Step 1: Set $S P=\sqrt{R}$ where $R$ represents total number of nodes, and choose the nodes with minimum ratio according to Eq. (28), Choose $K$ points and assigns vehicle to them and remove these points from NV,

Step 2: Start from the first vehicle,

Step 3: Update Eq. (28) for all remaining nodes located in NV, and randomly assigns some vehicles to the first node by considering the capacity,

Step 4: Remove the visited node as well as other neighbors, which are visited by this vehicle, update Eq. (28) and go to step 2,

Step 5: If the capacity of the vehicle is complete, choose another vehicle,

Step 6: If NV is complete, ends the algorithm, otherwise go to step 2.

\subsection{Parallel operations}

The parallel operation requires the following steps, 
Step 0: Assign all nodes, which are not visited by any vehicle to NV,

Step 1: Consider all vehicles,

Step 2: For all vehicles, update Eq. (28) for all nodes,

Step 3: Set $S P=\sqrt{R}$ where $R$ represents total number of nodes, and choose the nodes with minimum ratio according to Eq. (28), Choose one point for each vehicle and assign only the node with minimum amount,

Step 4: For the visiting node and its neighbor, if demand is bigger than capacities of these node, remove them from NV and go to step 2, otherwise assign them to the node and its neighbor with possible capacity and remove it from $\mathrm{NV}$,

Step 5: If NV is complete, ends the algorithm, otherwise go to step 2.

\subsection{Shaking phase}

The primary objective of this phase is to make some big changes on the solution and this could be accomplished through two methods of changing point and coverage of the visited points.

\subsubsection{Moving point}

Moving point strategy selects two vehicle and changes the routes and coverages of these two vehicles. Fig 3 shows a sample of this change.

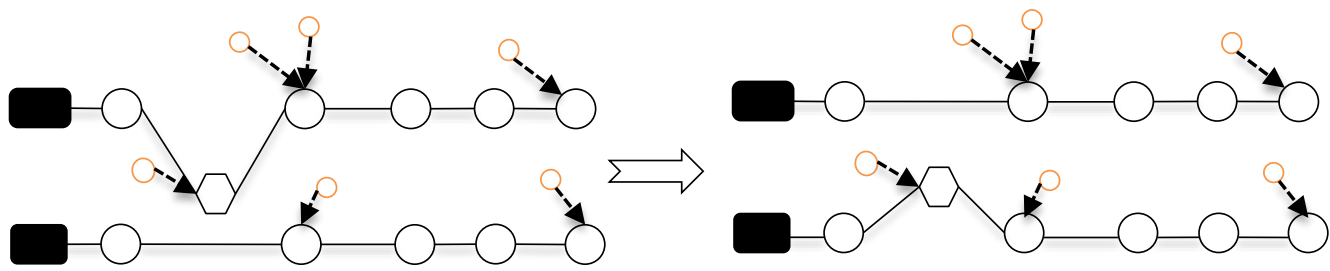

Fig. 3. Moving change

In Fig. 3, first $l_{1}=1,2,3$ and $l_{2}=1,2,3$ are chosen and four possible exchanges of $\left(l_{1}=1, l_{2}=0\right)$, $\left(l_{1}=1, l_{2}=1\right),\left(l_{1}=1, l_{2}=2\right)$ and $\left(l_{1}=3, l_{2}=2\right)$ are chosen for exchange.

\subsubsection{Changing covering and not covering points}

In this strategy, a meeting point in a vehicle is selected from a tour and if there are some points crossings the tour and they are within the range, the points are covered by the best possible point, otherwise, no change is applied. Table 1 shows details of change and notations.

\section{Table 1}

Different possible change in shaking phase

\begin{tabular}{cl}
\hline $\mathrm{K}$ & The method for creating neighborhood \\
\hline 1 & Covering the meeting point \\
2 & Changing the covering and not-covering point \\
3 & Changing one point with one point $\left(l_{1}=1, l_{2}=1\right)$ \\
5 & Changing one point with two points $\left(l_{1}=1, l_{2}=2\right)$ \\
6 & Changing two points with two points $\left(l_{1}=2, l_{2}=2\right)$ \\
\hline
\end{tabular}




\subsubsection{Local Search}

Local searches improve the quality of solutions and in this paper, we use two methods; change point and 2-opt algorithm. The first method simply considers all possible choices and selects the possible choice. The second method is executed either randomly or optimally. Consider a tour $j \in\{1, \ldots, \mathrm{i}, \mathrm{i}+1, \ldots, \mathrm{j}, \mathrm{j}+1, \ldots, n\}$ where $(i, i+1),(j, j+1)$ is removed and $(i, j),(i+1, j+1)$ is added. In optimal process, all possible choices of $i \in\{1,2, \ldots, n-2\}$ and $j \in\{i+2, \ldots, n\}$ are considered and the best choice is selected while in 2-opt we choose two positions of $i$ and $j$ randomly and execute the replacement. Fig. 4 demonstrates the structure of a sample of 2-opt change.

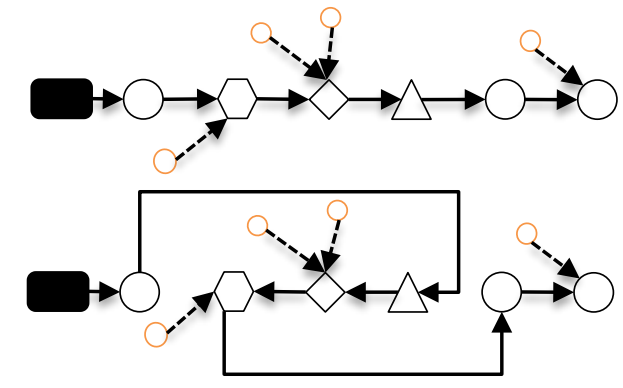

Fig. 4. The structure of 2-opt

We use three types of VNS; namely VNSI, VNSII and VNSIII. VNSI first applies shaking phase and after choosing the points, which have been changed, the algorithm performs 2-Opt on them to improve the performance. In VNSII, we apply local search on two changed points and finally, in VNSIII, the first best improvement is chosen. There is also another method named Adaptive VNS, which is similar to VNSI with the difference that shaking phase is executed based on special pattern. In this research, shaking phase is applied in special way that shaking steps given in Table 1 are not executed in order shown in the table and to generate neighborhood search, we use roulette wheel method. Let $P_{k}$ be the possible of choosing Kth method given in Table 1 and $W_{k}$ be the weight given for the improvement of method $k$. Therefore, we have

$$
P_{k}=W_{k} / \sum_{i} W_{i}
$$

The following summarizes the method.

1. Define the neighborhood structures $N \kappa$ with $K=1, . ., K \max$

2. Generate initial solution $S$

3. Improve initial solution by local search

4. $\mathrm{K} \leftarrow 1$

4. repeat

5. \{Adaptive Shaking\}

6. Select shaking method and generate $S_{-} \in N \kappa(S)$

7. $\{$ Local Search\}

8. $S^{*} \leftarrow \operatorname{localSearch}\left(S_{-}\right)$

9. \{Acceptance Decision\}

10. if $\mathrm{f}\left(S^{*}\right)<\mathrm{f}(\mathrm{S})$ then

11. $S \leftarrow S^{*}$

$K \leftarrow 1$

12. else

13. $\quad K \leftarrow K+1$

14. end if

15. Update weights and probabilities of shaking methods

16. until $\mathrm{K}<=\mathrm{Kmax}$ 


\section{Results}

In order to measure the performance of the proposed method, we generate several test problems in different sizes of small scales [ $10 \times 10$ ] and large scales [ $50 \times 50$ ] . Demand is a fuzzy number with lower limit of $U(3,8)$, middle distribution of $U(1,5)$ and upper limit of $U(1,6)$. The capacity of trucks is designed based on 0.85 of upper limits of trucks. The coverage ratio for small problems is between 0.2 and 0.4 and for large scale problems is $0.2 \pm 0.01$. In addition, between five to ten percent of the points are not eligible for establishing a depot. Table 2 demonstrates the parameters used for different test problems.

Table 2

The summary of test problems

\begin{tabular}{|c|c|c|c|c|c|c|}
\hline Test Problem & $\mathrm{R}$ & $\mathrm{n}$ & K & $\mathrm{Q}$ & $\mathrm{dis}_{\max }$ & $\rho$ \\
\hline 1 & 5 & 4 & 3 & 15 & 4 & 0.17 \\
\hline 2 & 6 & 5 & 3 & 19 & 6 & 0.33 \\
\hline 3 & 8 & 6 & 3 & 24 & 6 & 0.21 \\
\hline 4 & 9 & 7 & 3 & 30 & 9 & 0.43 \\
\hline 5 & 10 & 7 & 3 & 27 & 8 & 0.28 \\
\hline 6 & 10 & 9 & 3 & 35 & 7 & 0.30 \\
\hline 7 & 11 & 7 & 3 & 34 & 5 & 0.14 \\
\hline 8 & 12 & 8 & 3 & 30 & 8 & 0.36 \\
\hline 9 & 13 & 8 & 3 & 38 & 6 & 0.21 \\
\hline 10 & 13 & 9 & 3 & 36 & 7 & 0.25 \\
\hline 11 & 20 & 15 & 3 & 125 & 17 & 0.27 \\
\hline 12 & 30 & 25 & 3 & 210 & 15 & 0.25 \\
\hline 13 & 40 & 35 & 3 & 290 & 17 & 0.26 \\
\hline 14 & 45 & 40 & 5 & 200 & 16 & 0.25 \\
\hline 15 & 50 & 45 & 5 & 225 & 15 & 0.26 \\
\hline
\end{tabular}

Next, for each test problem given in Table 2, we provide the optimal solutions for different credit demand using CPLEX software package.

Table 3

The summary of simulation

\begin{tabular}{|c|c|c|c|c|c|c|c|c|c|c|c|c|}
\hline Problem & $\mathrm{Cr}$ & 0 & 0.1 & 0.2 & 0.3 & 0.4 & 0.5 & 0.6 & 0.7 & 0.8 & 0.9 & 1 \\
\hline \multirow{2}{*}{$\mathrm{P} 1$} & B & 14.9 & 14.9 & 14.9 & 14.9 & 13.7 & 13.7 & 13.7 & 13.7 & 13.7 & 13.7 & 13.7 \\
\hline & M & 14.9 & 20.5 & 18.8 & 18.4 & 17.1 & 19.4 & 19.5 & 20.3 & 21.8 & 22 & 22.5 \\
\hline \multirow{2}{*}{$\mathrm{P} 2$} & B & 29.6 & 29.6 & 26.9 & 24.8 & 24.8 & 24.8 & 24.8 & 24.8 & 24.8 & 24.8 & 24.8 \\
\hline & $\mathrm{M}$ & 29.6 & 74.4 & 74.4 & 74.4 & 24.8 & 24.8 & 51.5 & 52.5 & 74.4 & 74.4 & 74.4 \\
\hline \multirow{2}{*}{ P3 } & B & 29.3 & 29.3 & 24.8 & 24.8 & 24.8 & 24.8 & 24.8 & 24.8 & 24.8 & 24.8 & 24.8 \\
\hline & $\mathrm{M}$ & 29.3 & 74.4 & 74.4 & 27.5 & 42.7 & 52.5 & 56.1 & 74.4 & 74.4 & 74.4 & 74.4 \\
\hline \multirow{2}{*}{ P4 } & B & 21.2 & 18.4 & 18.4 & 18.4 & 18.4 & 18.4 & 18.4 & 18.4 & 18.4 & 18.4 & 18.4 \\
\hline & $\mathrm{M}$ & 21.2 & 18.4 & 18.4 & 18.4 & 18.4 & 18.4 & 18.4 & 18.4 & 36 & 42.7 & 42.7 \\
\hline \multirow{2}{*}{ P5 } & B & 35.4 & 31.8 & 31.8 & 31.8 & 29.1 & 28.6 & 28.6 & 28.6 & 28.6 & 28.6 & 28.6 \\
\hline & $\mathrm{M}$ & 35.4 & 41.5 & 41.4 & 41.4 & 41.4 & 44.4 & 41.5 & 41.5 & 41.5 & 41.5 & 41.5 \\
\hline \multirow{2}{*}{ P6 } & B & 24.9 & 18.4 & 18.4 & 18.4 & 18.4 & 18.4 & 18.4 & 18.4 & 18.4 & 18.4 & 18.4 \\
\hline & $\mathrm{M}$ & 24.9 & 55.2 & 54.8 & 18.5 & 18.5 & 18.4 & 18.5 & 44.2 & 55 & 55 & 55.1 \\
\hline \multirow{2}{*}{ P7 } & B & 52.8 & 22.9 & 19.7 & 19.7 & 18.4 & 18.4 & 18.4 & 18.4 & 18.4 & 16.5 & 15.2 \\
\hline & $\mathrm{M}$ & 52.8 & 58.2 & 19.7 & 22.7 & 45.6 & 45.6 & 49.9 & 50 & 50 & 55.1 & 60.5 \\
\hline \multirow{2}{*}{ P8 } & B & 24.7 & 24.7 & 24.7 & 24.7 & 24.7 & 24.7 & 24.7 & 18.6 & 18.6 & 18.6 & 18.6 \\
\hline & $\mathrm{M}$ & 24.7 & 24.7 & 24.7 & 24.7 & 24.7 & 24.7 & 24.7 & 49.3 & 51.3 & 55.8 & 59.4 \\
\hline \multirow{2}{*}{ P9 } & B & 52.8 & 28.9 & 19.7 & 19 & 18.6 & 18.4 & 18.4 & 18.4 & 18.4 & 16.5 & 15.2 \\
\hline & $\mathrm{M}$ & 52.8 & 49.6 & 65.3 & 64.6 & 68.5 & 68.3 & 68.3 & 73.4 & 69.4 & 74.7 & 76.7 \\
\hline \multirow{2}{*}{ P10 } & B & 18.6 & 18.6 & 18.6 & 18.6 & 18.4 & 18.4 & 18.4 & 18.4 & 18.4 & 18.4 & 18.4 \\
\hline & $\mathrm{M}$ & 18.6 & 50.5 & 49.9 & 48.3 & 20.2 & 45.6 & 48.7 & 49.9 & 51 & 50.1 & 51.3 \\
\hline \multirow{2}{*}{$\mathrm{P} 1$} & B & 64.3 & 70.7 & 59.1 & 42.8 & 21.5 & 23.4 & 23.4 & 26.5 & 31.6 & 32.3 & 35 \\
\hline & $\mathrm{M}$ & 64.3 & 75 & 73.1 & 53.8 & 21.5 & 29.4 & 35.4 & 49.5 & 61.6 & 70.1 & 121.7 \\
\hline \multirow{2}{*}{$\mathrm{P} 2$} & B & 124.6 & 124.6 & 123.8 & 100.8 & 95 & 86.5 & 79.7 & 79.6 & 72.3 & 59.3 & 59.9 \\
\hline & $\mathrm{M}$ & 124.6 & 124.6 & 129.7 & 103.5 & 97.3 & 88.3 & 89.6 & 96.5 & 100 & 130.8 & 126.3 \\
\hline \multirow{2}{*}{ P3 } & B & 155.1 & 146.8 & 135.6 & 121.9 & 100.6 & 100.2 & 98.3 & 78.4 & 77.5 & 58 & 112 \\
\hline & $\mathrm{M}$ & 155.1 & 150.6 & 135.6 & 140.4 & 100.9 & 105.2 & 100.2 & 98.3 & 100.6 & 138.1 & 152 \\
\hline \multirow{2}{*}{ P4 } & B & 148.6 & 144.2 & 138.2 & 130.8 & 115.7 & 105.6 & 101 & 105.5 & 104 & 97.6 & 94.2 \\
\hline & $\mathrm{M}$ & 148.6 & 144.2 & 138.2 & 140.4 & 121 & 140.5 & 150.3 & 139.2 & 140.6 & 152 & 155.1 \\
\hline \multirow{2}{*}{ P5 } & B & 106.2 & 99.6 & 103 & 91.9 & 103.1 & 91.1 & 104.2 & 107.4 & 102.8 & 92.3 & 82.2 \\
\hline & $\mathrm{M}$ & 106.2 & 99.6 & 103 & 91.9 & 103.1 & 91.1 & 150.1 & 169.3 & 174.2 & 180.2 & 180.7 \\
\hline \multicolumn{2}{|l|}{ AVERAGE } & 60.2 & 70.8 & 68.1 & 59.3 & 51 & 54.4 & 61.5 & 68.4 & 73.4 & 81.1 & 86.3 \\
\hline
\end{tabular}


The actual objective function is estimated and based on the failure occurs, the problem is repeated 1000 times and the mean of these 1000 times is measured and the optimal credit level is determined based on the measured means. Table 3 shows the results where $\mathrm{B}$ denotes the planned demands and $\mathrm{M}$ represents objective function resulted from simulation procedure.

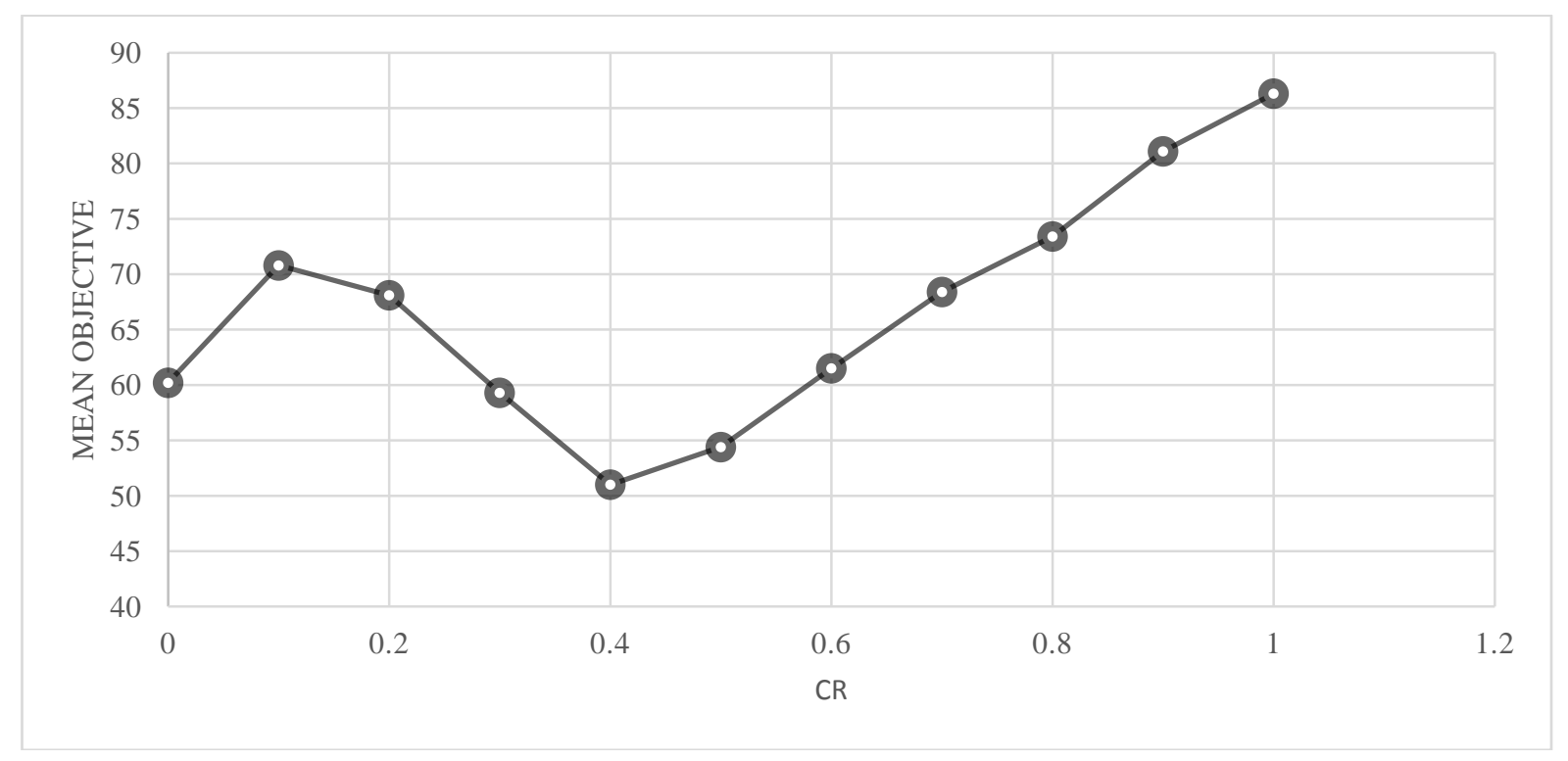

Fig. 5. The summary of mean of objective function for small sized problems

As we can observe from the results of Fig. 5, CR value increases as the mean of the objective function increases. Moreover, Table 5 demonstrates the results of the gap between different methods.

\section{Table 5}

The results of the proposed model for small scale problems

\begin{tabular}{|c|c|c|c|c|c|c|c|c|c|c|c|c|c|c|}
\hline \multirow{2}{*}{ PROBLEM } & \multicolumn{2}{|c|}{ CPLEX } & \multicolumn{3}{|c|}{ VNS I } & \multicolumn{3}{|c|}{ VNS II } & \multicolumn{3}{|c|}{ VNS III } & \multicolumn{3}{|c|}{ AVNS } \\
\hline & $\mathrm{B}$ & $\mathrm{T}$ & $\mathrm{B}$ & $\mathrm{T}$ & GAP & B & $\mathrm{T}$ & GAP & B & $\mathrm{T}$ & GAP & B & $\mathrm{T}$ & GAP \\
\hline P1 & 16.6 & 0.61 & 16.6 & 4.9 & 0 & 16.6 & 4.39 & 0 & 16.6 & 4.46 & 0 & 16.6 & 4.46 & 0 \\
\hline P2 & 16.6 & 0.83 & 16.6 & 6.2 & 0 & 16.6 & 4.34 & 0 & 16.6 & 4.49 & 0 & 16.6 & 4.49 & 0 \\
\hline P3 & 24.8 & 2.27 & 24.8 & 5.67 & 0 & 24.8 & 4.45 & 0 & 24.8 & 4.86 & 0 & 24.8 & 4.3 & 0 \\
\hline P4 & 24.8 & 4.2 & 24.8 & 5.37 & 0 & 24.8 & 4.77 & 0 & 24.8 & 5.23 & 0 & 24.8 & 5.5 & 0 \\
\hline P5 & 23.9 & 8.75 & 23.9 & 5.68 & 0 & 23.9 & 5.34 & 0 & 23.9 & 5.02 & 0 & 23.9 & 5.9 & 0 \\
\hline P6 & 18.5 & 12.45 & 18.5 & 6.4 & 0 & 18.5 & 4.69 & 0 & 18.5 & 5.45 & 0 & 18.5 & 6.4 & 0 \\
\hline P7 & 24.8 & 11.62 & 24.8 & 5.33 & 0 & 24.8 & 4.52 & 0 & 24.8 & 4.41 & 0 & 24.8 & 5.4 & 0 \\
\hline P8 & 18.4 & 84.39 & 18.4 & 10.63 & 0 & 19.2 & 10.27 & 4.17 & 19.2 & 11.75 & 4.17 & 18.9 & 12.9 & 2.65 \\
\hline P9 & 15.6 & 170.11 & 16.5 & 10.11 & 5.45 & 16.2 & 12.78 & 3.7 & 16.2 & 12.85 & 3.7 & 15.9 & 13.3 & 1.89 \\
\hline P10 & 18.6 & 3600 & 19.6 & 20.9 & 5.1 & 19.7 & 12.15 & 5.58 & 19.7 & 21.8 & 5.58 & 18.8 & 20.1 & 1.06 \\
\hline P11 & 30.6 & 3600 & 30 & 11.01 & 0 & 31.5 & 10.87 & 2.86 & 31.5 & 10.08 & 2.86 & 30 & 10.1 & 0 \\
\hline $\mathrm{P} 12$ & 42.22 & 3600 & 43.5 & 22.71 & 2.94 & 43.7 & 20.39 & 3.39 & 44.9 & 13.94 & 5.97 & 43.5 & 15.4 & 2.94 \\
\hline AVERAGE & 22.952 & 924.603 & 23.167 & 9.576 & 1.124 & 23.358 & 8.247 & 1.642 & 23.458 & 8.695 & 1.857 & 23.092 & 9.021 & 0.712 \\
\hline
\end{tabular}

The results presented in Table 5 are given in seconds and GAP represents the relative gap between metaheuristic and actual solver. According to the results, CPLEX could solve only 9 problems in less than one hour and for three problems, it can only find a feasible solution. For four scenarios, final error was less than $0.7 \%$ for AVNS, $1.1 \%$ for VNSI and the second scenario has performed the best among all possible four methods in terms of time. Fig. 6 shows the summary of CPU time for four methods. Table 6 presents the results of the implementation of the proposed methods for large scale problems. 


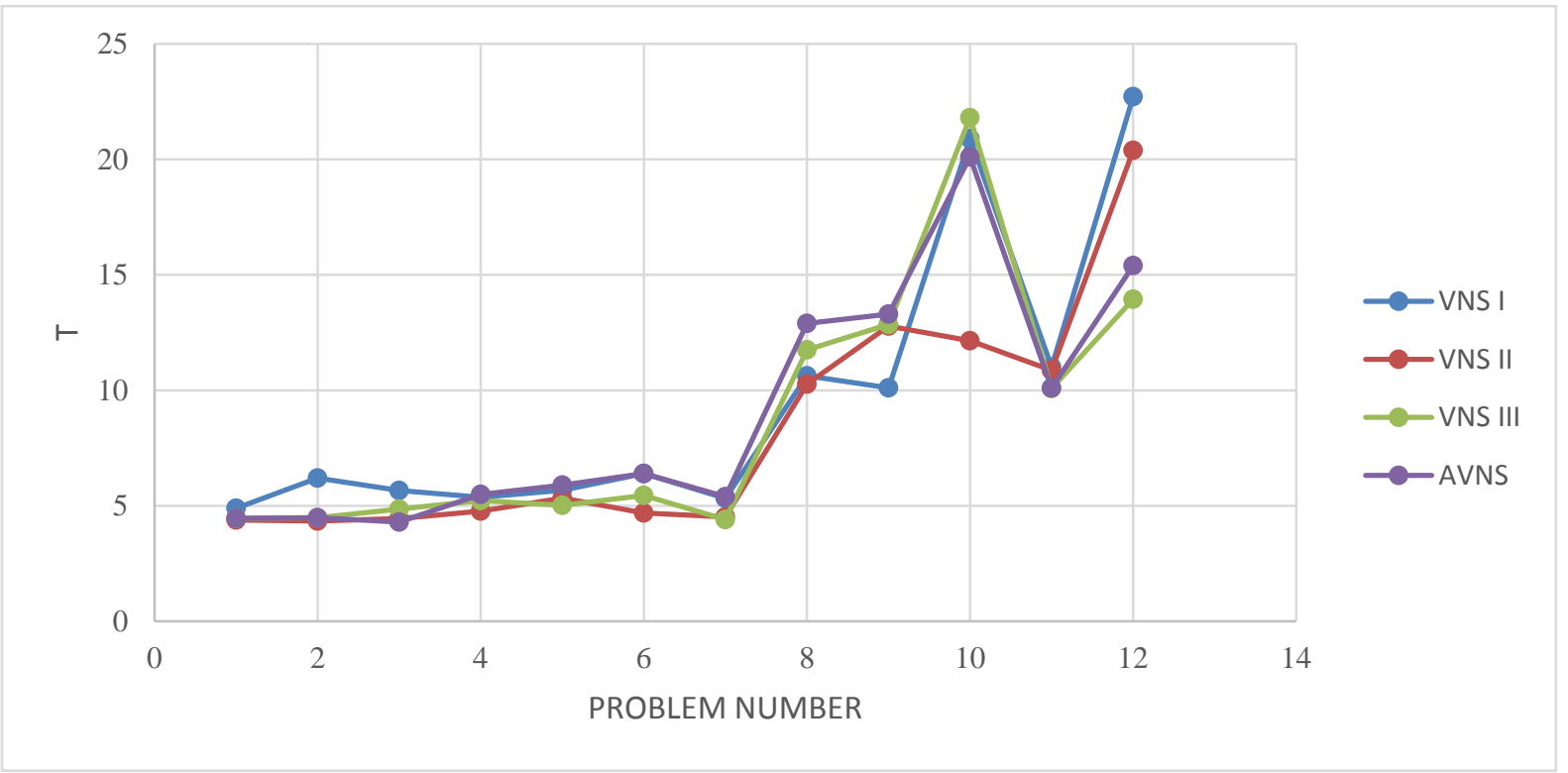

Fig. 6. The summary of CPU time for four methods

Table 6

The summary of the results of the proposed methods for large scale problems

\begin{tabular}{|c|c|c|c|c|c|c|c|c|c|c|c|c|c|c|c|c|c|}
\hline \multicolumn{2}{|c|}{ PROBLEM } & 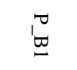 & '্ত্ & 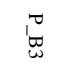 & $\left.\right|_{+} ^{\nabla}$ & '্ত্ & 官 & ఠ্ & 'ర & 'ه & '. & 吕 & $\underset{\sim}{\sigma}$ & $\underset{\omega}{\stackrel{\sigma}{\sigma}}$ & $\underset{\square}{\square}$ & 焉 & Mean \\
\hline \multirow{3}{*}{ VNS I } & B & 200.93 & 85.11 & 105.91 & 62.95 & 268.57 & 103.85 & 123.61 & 142.53 & 156.43 & 177.73 & 173.92 & 193.42 & 190.52 & 286.66 & 278.05 & \\
\hline & $\mathrm{AV}$ & 203.76 & 112.84 & 112.48 & 89.57 & 279.86 & 150.32 & 146.85 & 152.91 & 200.17 & 202.72 & 186.75 & 214.35 & 234.58 & 309.92 & 299.11 & \\
\hline & $\mathrm{T}$ & 6.28 & 6.95 & 10.77 & 9.88 & 9.54 & 13.06 & 23.38 & 18.93 & 28.82 & 22.91 & 30.43 & 31.73 & 35.09 & 41.2 & 75.52 & 24.299 \\
\hline \multicolumn{2}{|c|}{ GAP Best (\%) } & 0.2 & 5.8 & 6.5 & 0 & 5.8 & 0 & 1.9 & 5.8 & 0.4 & 3.5 & 0 & 2.1 & 7.4 & 2.0 & 0 & 2.7 \\
\hline \multicolumn{2}{|c|}{ GAP Average (\%) } & 0 & 9.0 & 4.0 & 0.1 & 3.3 & 6.2 & 4.9 & 2.4 & 6.3 & 6.5 & 0 & 0 & 6.3 & 0 & 0 & 3.3 \\
\hline \multirow{3}{*}{ VNS II } & B & 207.43 & 80.63 & 98.93 & 64.85 & 252.96 & 111.33 & 123.61 & 134.91 & 163.77 & 183.17 & 176.52 & 189.28 & 191.16 & 295.68 & 301.94 & \\
\hline & $\mathrm{AV}$ & 208.69 & 109.47 & 107.9 & 90.35 & 279.9 & 140.89 & 139.62 & 151.61 & 195.63 & 189.39 & 193.62 & 216.82 & 232.64 & 324.25 & 326.74 & \\
\hline & $\mathrm{T}$ & 5.73 & 6.57 & 8.09 & 8.79 & 8.8 & 17.16 & 14.06 & 15.99 & 17.13 & 21.18 & 21.27 & 31.03 & 30.45 & 40.65 & 71.64 & 21.236 \\
\hline \multicolumn{2}{|c|}{ GAP Best (\%) } & 3.3 & 0.5 & 0 & 2.9 & 0 & 6.7 & 1.9 & 0.5 & 4.9 & 6.4 & 1.4 & 0 & 7.7 & 5 & 7.9 & 3.2 \\
\hline \multicolumn{2}{|c|}{ GAP Average (\%) } & 2.3 & 6.2 & 0 & 1.0 & 3.3 & 0 & 0 & 1.6 & 4.2 & 0 & 3.5 & 1.1 & 5.5 & 4.4 & 8.4 & 2.8 \\
\hline \multirow{3}{*}{ VNS III } & B & 200.93 & 84.63 & 100.16 & 67.46 & 252.96 & 110.85 & 121.25 & 134.2 & 159.54 & 171.44 & 179.04 & 199.33 & 181.93 & 293.89 & 294.95 & \\
\hline & $\mathrm{AV}$ & 207.37 & 106.46 & 112.84 & 89.42 & 272.79 & 146.94 & 145.51 & 149.18 & 187.39 & 193.97 & 200.06 & 219.32 & 219.63 & 316.46 & 320.88 & \\
\hline & $\mathrm{T}$ & 5.68 & 6.53 & 8.11 & 8.76 & 9.4 & 16.41 & 14.39 & 15.23 & 16.53 & 16.1 & 25.04 & 30.32 & 25.78 & 39.88 & 68.83 & 20.466 \\
\hline \multicolumn{2}{|c|}{ GAP Best (\%) } & 0.2 & 5.2 & 1.2 & 6.6 & 0 & 6.3 & 0 & 0 & 2.3 & 0 & 2.8 & 5.0 & 3.0 & 4.4 & 5.7 & 2.8 \\
\hline \multicolumn{2}{|c|}{ GAP Average (\%) } & 1.7 & 3.6 & 4.3 & 0 & 0.8 & 4.1 & 4.0 & 0 & 0 & 2.3 & 6.6 & 2.2 & 0 & 2.0 & 6.7 & 2.5 \\
\hline \multirow{3}{*}{ AVNS } & $\mathrm{V}$ & 200.51 & 80.16 & 98.98 & 65.23 & 260.29 & 111.18 & 121.25 & 135.4 & 155.74 & 180.28 & 181.98 & 200.88 & 176.34 & 280.91 & 290.06 & \\
\hline & $\mathrm{AV}$ & 203.76 & 102.61 & 110.27 & 93.54 & 270.41 & 145.8 & 150.26 & 154.12 & 189.53 & 192.39 & 194.49 & 224.84 & 221.17 & 310.99 & 307.33 & \\
\hline & $\mathrm{T}$ & 10.44 & 11.25 & 16.19 & 15.89 & 15.26 & 23.47 & 27.95 & 33.2 & 23.48 & 39.06 & 44.83 & 47.73 & 45.19 & 50.71 & 81.39 & 32.403 \\
\hline \multicolumn{2}{|c|}{ GAP Best (\%) } & 0 & 0 & 0.0 & 3.5 & 2.8 & 6.5 & 0 & 0.8 & 0 & 4.9 & 4.4 & 5.7 & 0 & 0 & 4.1 & 2.2 \\
\hline \multicolumn{2}{|c|}{ GAP Average (\%) } & 0 & 0 & 2.1 & 4.4 & 0 & 3.3 & 7.0 & 3.2 & 1.1 & 1.5 & 3.9 & 4.6 & 0.7 & 0.3 & 2.6 & 2.3 \\
\hline
\end{tabular}

As we can observe from the results of Table 6, AVNS has performed the best followed by VNSIII. Fig. 7 shows the results of CPU time used for each method.

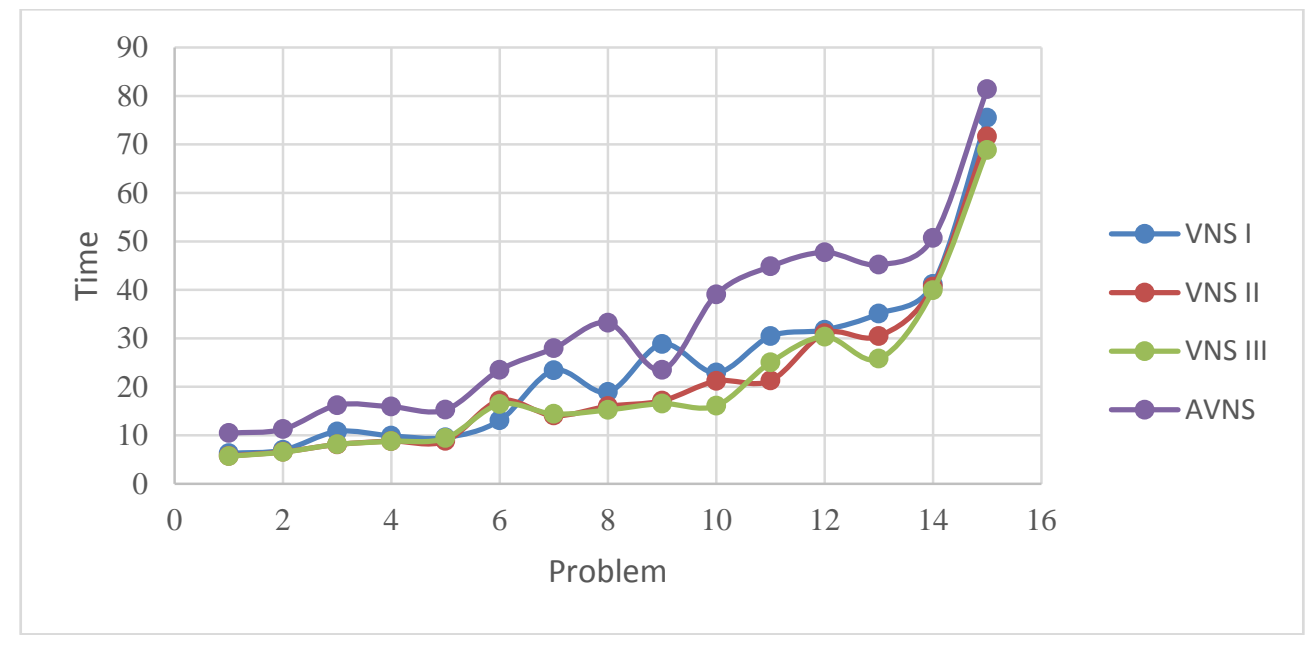

Fig. 7. The results of CPU time for the implementation of the proposed method 
As we can observe from the results of Fig. 7, VNS II and VNS III perform the best in terms of CPU time.

\section{Conclusion}

In the event of crises and catastrophes, time plays an essential role in development of providing humanitarian efforts. The number of casualties and damage increases dramatically as time increases when a natural disaster occurs. Therefore, the logistics activities must be provided in minimum amount of time to help rescue people as quickly as possible. In this study, we have provided a comprehensive model to aid people who face crisis. The proposed study presented a location and multi-depot vehicle routing for emergency vehicles using tour coverage and random sampling. The proposed model has been solved using some metaheuristics for some randomly selected problems in small and large scales. The preliminary results have indicated that the method could find good quality solutions in reasonable amount of time.

\section{Acknowledgement}

The authors would like to thank the anonymous referees for constructive comments on earlier version of this paper.

\section{References}

Abounacer, R., Rekik, M., \& Renaud, J. (2014). An exact solution approach for multi-objective location-transportation problem for disaster response. Computers \& Operations Research, 41, 8393.

Afshar, A., \& Haghani, A. (2012). Modeling integrated supply chain logistics in real-time large-scale disaster relief operations. Socio-Economic Planning Sciences, 46(4), 327-338.

Barbarosoğlu, G., Özdamar, L., \& Cevik, A. (2002). An interactive approach for hierarchical analysis of helicopter logistics in disaster relief operations. European Journal of Operational Research, 140(1), 118-133.

Cao, E., \& Lai, M. (2010). The open vehicle routing problem with fuzzy demands. Expert Systems with Applications, 37(3), 2405-2411.

Croes, G. A. (1958). A method for solving traveling-salesman problems. Operations Research, 6(6), 791-812.

Doerner, K., Focke, A., \& Gutjahr, W. J. (2007). Multicriteria tour planning for mobile healthcare facilities in a developing country. European Journal of Operational Research, 179(3), 1078-1096.

Feo, T. A., \& Resende, M. G. (1989). A probabilistic heuristic for a computationally difficult set covering problem. Operations research letters, 8(2), 67-71.

Fleszar, K., Osman, I. H., \& Hindi, K. S. (2009). A variable neighbourhood search algorithm for the open vehicle routing problem. European Journal of Operational Research, 195(3), 803-809.

Ghaffari-Nasab, N., Ahari, S. G., \& Ghazanfari, M. (2013). A hybrid simulated annealing based heuristic for solving the location-routing problem with fuzzy demands. Scientia Iranica, 20(3), 919930.

Kaufmann, A. (1975). Introduction to the theory of fuzzy subsets (Vol. 1, No. 8). New York: Academic Press.

Knott, R. (1987). The logistics of bulk relief supplies. Disasters, 11(2), 113-115.

Liu, B. (2004). Uncertainty Theory. An Introduction to Its Axiomatic Foundation, vol. 154 of. Studies in Fuzziness and Soft Computing.

Luis, E., Dolinskaya, I. S., \& Smilowitz, K. R. (2012). Disaster relief routing: Integrating research and practice. Socio-economic planning sciences, 46(1), 88-97.

Mladenović, N., \& Hansen, P. (1997). Variable neighborhood search. Computers \& Operations Research, 24(11), 1097-1100. 


\section{Appendix}

\section{Table 7}

The input parameters for small problem

\begin{tabular}{cccccccc}
\hline Problem & $\mathrm{R}$ & $\mathrm{n}$ & $\mathrm{K}$ & $\mathrm{Q}$ & dismax & $\rho$ & Ba \\
\hline P1 & 5 & 4 & 3 & 15 & 7 & 0.29 & 0 \\
P2 & 6 & 5 & 3 & 30 & 6 & 0.20 & 0 \\
P3 & 8 & 6 & 3 & 30 & 5 & 0.25 & 0 \\
P4 & 9 & 7 & 3 & 25 & 8 & 0.25 & 0 \\
P5 & 10 & 7 & 3 & 30 & 7 & 0.23 & 0 \\
\hline P6 & 11 & 9 & 3 & 40 & 7 & $0 / 25$ & 0 \\
P7 & 11 & 7 & 3 & 35 & 7 & 0.27 & 1 \\
P8 & 13 & 10 & 3 & 50 & 8 & 0.27 & 2 \\
P9 & 15 & 10 & 3 & 45 & 9 & 0.35 & 2 \\
\hline P10 & 20 & 15 & 3 & 125 & 2 & 0.1 & 1 \\
\hline P11 & 20 & 15 & 3 & 125 & 7 & 0.27 & 3 \\
\hline P12 & 25 & 15 & 3 & 125 & 7 & 0.27 & 3 \\
\hline
\end{tabular}


Table 8

The results of large scale problem

\begin{tabular}{|c|c|c|c|c|c|c|}
\hline Problem & $\mathrm{R}$ & $\mathrm{n}$ & $\mathrm{K}$ & Q & dismax & $\mathrm{Ba}$ \\
\hline B_P1 & 15 & 12 & 3 & 86 & 21 & 3 \\
\hline B_P2 & 20 & 15 & 3 & 119 & 21 & 3 \\
\hline B_P3 & 25 & 22 & 5 & 103 & 23 & 3 \\
\hline B_P4 & 30 & 25 & 5 & 120 & 20 & 4 \\
\hline B_P5 & 35 & 20 & 5 & 95 & 20 & 4 \\
\hline B_P6 & 40 & 35 & 5 & 166 & 23 & 4 \\
\hline B_P7 & 50 & 40 & 8 & 119 & 21 & 5 \\
\hline B_P8 & 60 & 50 & 8 & 148 & 23 & 6 \\
\hline B_P9 & 70 & 55 & 8 & 156 & 23 & 6 \\
\hline B_P10 & 75 & 65 & 8 & 210 & 22 & 6 \\
\hline B_P11 & 80 & 70 & 10 & 163 & 22 & 7 \\
\hline B_P12 & 90 & 80 & 10 & 183 & 23 & 8 \\
\hline B_P13 & 100 & 90 & 10 & 208 & 22 & 10 \\
\hline B_P14 & 150 & 130 & 10 & 296 & 23 & 15 \\
\hline B_P15 & 200 & 160 & 20 & 187 & 23 & 20 \\
\hline
\end{tabular}

